\title{
RELEVANZGESTEUERTER MORPHOLOGISCHER UMBAU IM FRÜHNEUHOCHDEUTSCHEN
}

\section{Einleitung}

Das Frühneuhochdeutsche hat ohne Zweifel als die morphologisch aktivste und dynamischste Periode der deutschen Sprachgeschichte zu gelten. Die morphologischen Umstrukturierungen, Neuordnungen und Rationalisierungen wirken bis heute nach. Dagegen sind die vorherigen Perioden Alt- und Mittelhochdeutsch primär durch phonologische Umbrüche gekennzeichnet: Einerseits durch zwei große Umlautphasen, bei denen Merkmale unbetonter Vokale regressiv auf den betonten Vokalismus projiziert wurden, andererseits durch Schwächung und Abbau nichtbetonter Vokale. Dieser phonologische Wandel hat das morphologische System massiv affiziert - nicht nur insofern, als suffigierend realisierte Kategorien in ihrer Realisierung bedroht waren, sondern indem auch sVerzerrungen * stattgefunden haben. So wurden durch den anfänglich rein phonetisch motivierten Umlaut Informationen, die bis dato nur in der Wortperipherie ausgedrückt wurden, sautomatisch « in die Wurzel befördert - etwa die Kategorie sKasus (Genitiv und Dativ im Singular) und 'Numerus` (Plural) bei Substantiven oder die Kategorie ,Modus (Konjunktiv) beim starken Verb. Wahrend manche dieser phonologischen "Angebote $*$ in den folgenden Jahrhunderten von der Morphologie aufgegriffen und grammatikalisiert wurden, hat sie andere abgelehnt: Kasusumlaute beim Substantiv wurden in der sog. ersten (ahd.) und zweiten (mhd.) sparadigmatischen Ausscheidung von Umlautvariantene (Sonderegger 1979, S. 308-310) per Analogie schon bald wieder aus der Wurzel beseitigt, Plural- und Konjunktivumlaute haben sich dagegen bis heute gehalten bzw. wurden sogar ausgebaut. Die Morphologie reagiert schnell auf phonologischen Wandel und keineswegs erst dann, wenn sie in ihrem Bestand bedroht ist. Beim Substantiv besteht das wichtigste morphologische Ziel in der Numerusprofilierung, beim Verb in der Tempusprofilierung.

Dieser Beitrag befaßt sich ausschließlich mit dem Verbalbereich und hat zum Ziel, der inneren Struktur und Ratio des Umbaus näherzukommen und diese anhand des in Bybee (1985) und (1994) entwickelten Relevanzkonzepts theoretisch zu fundieren: Nicht nur die Prinzipien dieses Umbaus, auch die Chronologie seiner Einzelschritte folgt genau den von Bybee durch synchronen Sprachvergleich ermittelten kategoriellen Rele- 
vanzgraden. Die im Spätmhd. zerruttete und sverzerrte- Morphologie wird - gestevert durch die jeweilige Kategorienrelevanz - entzerrt, justiert, hierarchisiert und systematisiert. Dabei kristallisieren sich die bekannte Tempusprofilierung, daneben auch eine Modusprofilierung heraus, nicht jedoch eine Aspektprofilierung, wiewohl Anstitze zu einer solchen Grammatikalisierung vorhanden waren. Diese wurden jedoch nivelliert wie zu zeigen sein wird, zugunsten einer stärkeren Tempusprofilierung. In diesem Punkt unterscheidet sich das Deutsche von den meisten germanischen Sprachen.

\section{Zum Relevanzkonzept von Bybee (1985)}

50 Sprachen, die weder genetisch noch areal zueinander in Beziehung stehen, ${ }^{1}$ bilden die Grundlage fur Bybees Untersuchung zu der Frage, welche Informationen bzw. Kategorien auf welche Weise ausgedruickt werden, d.h. wie sich genau das Funktion/Form-Verhaltnis gestaltet.

\subsection{Form: Die Skala der fünf Ausdrucksverfahren}

Je nach dem Grad morphologischer Fusionierung gelangt Bybee zu folgender Skala prinzipiell mōglicher Ausdrucksverfahren:

lexikalisch - derivationell - flexivisch - klitisch/frei grammat. - syntaktisch

\section{Zunehmender Fusionsgrad}

Figur 1: Die wichtigsten Ausdrucksverfahren (nach Bybee 1985, S. 12)

Lexikalisch realisierte Informationen sind untrennbar in einem Lexem miteinander verschmolzen, z. B. Auto, während am entgegengesetzten Pol mit dem syntaktischen Verfahren die maximale Expandienung der Informationen erfolgt (vom Menschen gesteuertes, motorisiertes Gefahrt mit vier Radem). Dazwischen liegen die Verfahren klitisch, flexivisch und derina. tionell. Klitika sind phonologisch verschmolzene Syntagmen, die in aller Regel noch aunosbar sind, während Flexion die obligatorische Kombination einer Wurzel mit Flexiven vorsieht. Reihenbildung. Produktivitat und Bedeutungskonstanz sind hier hochgradig vorhanden - im Gegensatz zur Derivation, wo Blockaden und damit mangelnde Reihenbildung ('Diebchen) sowie Lexikalisierungen mehr oder weniger ausgeprägt vorkommen (Hermenen *,kleiner Herr, sondern 'Hundehalter).

\footnotetext{
1. Fur die indoeuropaische Familie wurde das Serbische gewahit; au den 50 Spra-
} chen s. die Liste in Bybee (1985, S. 2151.).
Welche Informationen mit welchem Fusionsgrad realisiert werden, hängt von deren Semantik, genauer von deren Relevanz bezuglich der Wurzel ab.

2.2 Funktion: Welche Informationen werden wie und wo am Verb ausgedrückt?

\subsubsection{Relevanz}

Relevanz wird von Bybee wie folgt definiert:

-A meaning element is relevant to another meaning element if the semantic content of the first direetly affects or modifies the semantic content of the second. If two meaning elements are, by their content, highly relevant to one another, then it is predicted that they may have lexical or inflectional expression, but if they are irrelevant to one another, then their combination will be restricted to syntactic expression. - (Bybee 1985, S. 13, Hervorhebung im Original)

Das Relevanzprinzip sieht also vor, daß mit zunehmendem Relevanzgrad zunehmende Fusionienung des Informationsausdrucks korreliert: =The degree of relevance predicts the likelihood of lexical or derivational expression of the grammatical category, the order of affixes with respect to the stem, and the degree of morphophonological fusion between the gram and the stem. - (Bybee et al. 1994, S. 22). Diese Relationen konstituieren diagrammatischen Ikonismus, der dem rein quantifizierenden Ikonis. muskonzept der Natïlichkeitstheorie entgegensteht. ${ }^{2}$ Bezüglich der Relevanzgrade gelangt Bybee zu folgender Hierarchie:

\section{+ relevant}

Valenz Diathese Aspelct Tempus Modus Numeris Person

Figur 2: Relevanzhierarchie verbaler Kategorier

Zu den valenzändernden Kategorien gehören z. B. Kausativa: Hier finden so tiefgreifende Veränderungen statt, daß diese Komplexe of lexika. lisch, z. T, auch derivationell realisiert werden; vgl, nhd. sterben/töten, stehen/stellen, sitzen/setzen, trinken/ränken etc. Die Diathese verändert die gesamte Richtung der Handlung: Im Aktiv markiert das Subjekt das Agens, im Passiv jedoch das Patiens, $\mathbf{d}$. h. hier werden ganze Rollen vertauscht, weshalb auch hier der lexikalisch/derivationelle Ausdruck dominiert (vgl. kaufen/benkaufen). Aspekt betriff den internen Verlauf der

\footnotetext{
Das naturfichkeitstheoretische Konzept des konstruktionellen Ikonismus basiert auf der Forderung, das einem .Mehrr auf semantischer Seite auch ein konstruk. tionelles iMehrv entsprechen sollte (vgl. Mayerthater 1081, S. $23 \mathrm{fr}$ ).
} 
Verbalhandlung, d.h. ihre Zerlegung in verschiedene Phasen (z. B. inchoativfingressiv, durativ, resultativ/perfeltiv etc.). Auch diese Information ist hochrelevant, da sie direkt in den Verlauf der durch das Verb bezeichneten Handlung eingreift. Anders Tempus, dem zwar auch ein hoher Relevanzgrad zukommt, das aber die durch das Verb bezeichnete Handlung nur extern temporal situiert, sie als solche jedoch sintakt- lät: Die Handlung wird nur in verschiedene Zeitstufen transponiert. Modus betriff in weitestem Sinn die Haltung des Sprechers zum bezeichneten Sachverhalt, d.h. zur gesamten Proposition. Modus veràdert weniger die Semantik des Verbs, sondem macht Aussagen über den Faktizitätsgrad der Proposition: über die vom Sprecher angenommene Wahrscheinlichkeit für das Eintreten des Sachverhalts, die Quelle, aus der er das Wissen bezieht; als Imperativ die Aufforderung zum Vollzug der Handlung etc. Wieder bleibt die Handlung als solche unberīhrt. Numerus und Person betreffen nur die Aktanten des Vertos und verädern nicht seine lexikalische Bedeutung Sie werden unter die sog. wagreement categoriese subsumiert, da sie sich weniger auf das Verb als auf (meist) das Subjekt beziehen, das ublicherweise durch die NP realisiert wird.

Nun werden diese unterschiedlich relevanten Informationen keines. wegs alle flexivisch realisiert. Bei der Frage, welche Art von Informationen flexivischen Ausdruck wahrscheinlich macht, ist das sog. Allgemeingültigkeitsprinzip zu berüicksichtigen.

\section{2 .2 Aligemeingoltigkeit}

Allgemeingültigkeit (ogenerality") definient sich gemäß Bybee (1985, S. 16f.) wie folgt:

"The second factor that needs to be taken into consideration in determining what can be an inflectional category is lexical generality. By definition, an infectional category must be applicable to all stems of the appropriate semantic and syntactic category and must obligatorily occur in the appropriate syntactic context. In order for a morphological process to be se aeneral, it must have only minimal semantic content. If a semantic element has high content, L.e. is very specific, it simply will not be applicable to a large number of stems.-

However, generality distinguishes inflectional from all the rest. Inflectional categories are more general - have a wider range of applicability with predictable meaning - than lexical, derivational, or periphrastic categories. Thus generality is a necessary defining feature of inflection. (Bybee et al. 1994, S. 22)

Flexionskategorien soltten aus einer möglichst allgemeinen Bedeutung mit begrenztem Inhalt bestehen. So ist die Person/Numerus-Kategorie von hoher Allgemeingultigkeit und, damit verbunden, hochgradig kompatibel mit fast allen Verben. Flexion gestaltet sich damit als eine Funktion zwi- schen hoher Relevanz und hoher Allgemeingultigkeit, d. h. auf Figur 3 bezogen befindet sich Flexion idealerweise im mittleren Oberschneidungsbereich zwischen relativ hoher Relevanz sowie hoher Allgemeingultigkeit: Einerseits muß die Information so relevant sein, daß sich ihr obligatorischer Ausdruck lohnt, andererseits darf sie nicht zu komplex und umfangreich sein. Nur dann ist gewährleistet, das sie sich mit jedem Mitglied einer (flektierenden) Wortart verbinden und dabei zu einer vorhersagharen Gesamtbedeutung fuhren kann, womit sie die zentralen Kriterien der Flexion (Reihenbildung und Bedeutungskonstanz) erfullt.

Die Auswertung von Bybees Korpus hat ergeben, daß die relevantesten Kategorien Valenz, Diathese und Aspekt nicht selten auch lexikalisch bzw. derivationell realisiert werden. Klar kristallisiert sich heraus, daß TAM, d.h. Tempus, Aspekt und Modus, die am weitesten verbreiteten Flexionskategorien sind: Aspekt wird $\mathrm{zu} 52 \%$ flexivisch realisiert. Tempus zu $48 \%$ und Modus sogar zu $68 \%$. Auch die minderrelevanten, aber sehr allgemeingultigen Kategorien Numerus (54\%) und Person (Subjekt) $(56 \%)$ bilden in vielen Sprachen Flexionskategorien. Zieht man das derivationelle Ausdrucksverfahren hinzu, so betrifft dieses vorrangig die sehr relevanten, doch wenig allgemeingültigen Kategorien Valenz, Diathese und Aspekt. Figur 3 visualisiert diese Zusammenhänge, wobei die in Frage stehenden Bereiche der Derivation und Flexion besonders in den Mittelpunkt geruickt werden. Das syntaktische und klitische Verfahren (nach Bybee sfree grammaticals) wurden zu ssyntaktisch = zusammengefast. Die einzelnen Kategorien wurden in den Bereichen sderivationell - flexivisch $=$ angeordnet; die Punkte deuten an, daß es weitere Ausdrucksmöglichkeiten gibt. Die erste der beiden hinzugefugten Prozentzahlen bezieht sich auf den derivationellen, die zweite auf den flexivischen Ausdruck.

+ fusionierend

+ relevant

- allgemeinguiting

+ frequent

viel Allomorphie

(lexikalisch) derivationell

- fusionierend - relevant + allgemeingultig

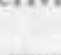$$
\text { Valenz }(84 \% 6 \%
$$$$
\text { Nan.... Diathese }(30 \% 0 \%)
$$$$
\text { _t...... Aspekt (22\%52\%) }
$$$$
\text { Tempus (2\%48\%) }
$$$$
\text { - Modus }(0 \$ 168 \%)
$$$$
\text { - Numerus }(12 \% 54 \%)
$$$$
\text { - Person (0\%56\%) }
$$

Figur 3: Relevanz, Allgemeingultigkeit und deren prototypische Realisierung 


\subsection{Gebrauchsfrequenz}

Eine zentrale Rolle kommt der Gebrauchsfrequenz za: Bybee macht immer wieder (z B. 1985, S. 119 ff.) deutlich, daB - unabhangig vom jeweiligen Relevanzgrad der Kategorie - die besonders häufige Verwendung einer bestimmten Wortform eine Linksdrift auf der Skala bewirkt. Dieses Prinzip hat sich in der Arbeit zu den Irregularisienungsstrategien bei Verben von Nubling (2000) immer wieder bestatigt: Irregularisierungen, die nichts anderes sind als zunehmende Fusionierung und Allomorphie, entwickeln sich besonders schnell und ausgeprägt bei hochfrequenten Verben. So wird der Person/Numenus-Ausdruck im Deutschen ublicherweise additiv-suffigierend realisiert (lach-e, lach-st, lach-t etc.). Im Fall häufiger verwendeter starker Verben hält sich bis heute die (mehr oder weniger lautgesetzlich entstandene) Wechselflexion, d.h. Hebung oder Umlaut (UL) des Wurzelvokals nur in der 2, und 3. Person $\mathrm{Sg}$. Prisens geb-e vs. gib-st/gib-t, fahr-e vs. fahr-st/fahr-t; s. Abschn. 4.1). Im Fall des häufigsten aller Verben, sein, werden diese beiden Kategorien lexikalisch-suppletiv realisiert: bin vs. bist vs. ist etc. (Personensuppletion). Im Fall von ist vs. war liegt Tempussuppletion vor, bei ist vs. sei Modussuppletion. In der Regel setzt der irreguläre bzw. suppletive Ausdruck weniger relevanter Kategorien ebenfalls irregulären bzw. suppletiven Ausdruck der hōherrelevanten Kategorien (links davon auf der Skala) voraus, wobei der Grad an Irregularität dabei meist noch zunimmt. Bef alledem spielt sowohl die lexikalische Frequenz des konkreten Verbs eine Rolle als auch die kategorielle Frequenz, d.h. im gegenüber dem Prateritum viel frequenteren Prasens sind fusionierende Person/Numerus-Ausdrücke weitaus wahrscheinlicher. Dabei haben sich Implikationen der folgenden Art ergeben: Erfolgt der Ausdruck einer Kategorie A in Kombination mit der weniger frequenten Auspraigung einer relevanteren Kategorie B auf irreguläre Weise, so gilt, daß Kategorie $\mathrm{A}$ in Verbindung mit der frequenteren Ausprägung von Kategorie B mindestens ebenso irregulär, wenn nicht irregulärer ausgedrilckt wird. So trifft das Englische im Singular des Prateritums ausschließlich von be eine Personenunterscheidung: (I) was, (you) were, (she) was (PL: were). Dies läßt auch mindestens eine entsprechende, wahrscheinlich sogar stärkere Personenunterscheidung im Präsensparadigma erwarten, was sogar in zweierlei Hinsicht zutrifft: Mit ( $T$ ) am, (you) are, (s/he) is wird nicht nur die zweifache Personendifferenzierung im Priteritum durch eine dreifache übertroffen, sondern die formale Unterschiedlichkeit, der inteme Differenzierungsgrad ist ausgepraigter. Besteht bei washoere =nur* schwache Suppletion, so sind am/are/is als stark suppletiv zu klassifizieren, wobei sich die frequenteste $3 . \mathrm{Sg}$. is am stärksten von den anderen Formen abhebt.

\subsection{Allomorphie}

Schließlich erweist sich auch das Auftreten von Allomorphie (morphologischer Synonymie) als relevanzgesteuert: Grundsaitzlich scheint zu gelten, daß mit dem Grad an Relevanz einer Kategorie das Ausmaß ihrer allomorphischen Realisierung korreliert - gleichzeitig mit ihrer Vorverlagerung in die Wurzel. Hier sei nur die Aspektallomorphie in slawischen Sprachen erwathnt oder die Ternpusallomorphie in Form von ca. 40 Vokalalternanzen bei den starken Verben des Deutschen.

In Bybee (1985) erfahrt man relativ wenig uber die genaue Ermittlung dieser anhand von 50 Sprachen erhobenen Werte. Ware das Deutsche berucksichtigt worden, so stellte sich z. B. die Frage, wo der Tempusausdruck auf der Ausdruckssikala verortet. wird, wenn man die Koexistenz. von (morphologisch realisiertem) Präsens und Praiteritum und (morphosyntaktisch realisiertem) Perfekt und Futur bedenkt. Kritische Distanz ist sicherlich angesichts der verwendeten Referenzwerke angebracht; manche wirken wenig professionell und sind oft viele Jahrzehnte alt. Muttersprachler ăuBem immer wieder Kritik an der Richtigkeit der präsentierten Sprachdaten bzw, an deren Interpretation. ${ }^{3}$ Eingedenk aller dieser Vorbehalte bestatigt sich jedoch die Gültigkeit der auf dieser breiten Basis gewonnenen Erkenntnisse. Ganz besondere Evidenz dafür liefert die deutsche Sprachgeschichte, genauer: der morphologische Umbau in der Periode des Fruhmeuhochdeutschen.

\section{Die fruhneuhochdeutsche Verbalflexion: Profilierung durch Nivellierung}

Die in den folgenden Abschnitten zu prisentierenden morphologischen Umbriche des Frühnhd. werden auf der faktischen Ebene als bekannt vorausgesetzt und daher immer nur kurz skizziert. ${ }^{4}$ Neu ist ihre Koppelung mit der Relevanzhierarchie von Bybee, was diesem Wandel eine klare Zielrichtung verleiht. Gezeigt werden soll zum einen, daß der morphologische Umbau zu einer Profilierung der relevanteren Kategorien Tempus und Modus fuhrt. Die Profilierung erfolgt dabei weniger durch exponierende Maß. nahmen innerhalb dieser beiden Kategorien als vielmehr durch Nivellie-

\footnotetext{
= Wenig vertrauenerweckend ist auch das folgende Zitat: - In general, in examining the grammans it was possible to follow the author's division of categories into derivational and inflectional, even if explicit criteria were not siven. it was asen. med that most linguists' intritive undenstanding of the infectional/derivational distinction coincides with the criteria made explicit hors, and the author, whe is the expert on the language, should be given the last word (Bytee 1805, S. 27)

is the cxpert on the tartgunge, should be given tie last word- (bybee 1885, S. 27). Zu den Daten s. 2 B. Erben (1970), Aubst (1975), MosenStopp (Higs) (1968), Ebert at al. (1933), WegeraSolms (2000).
} 
rungen der Nachbarkategorien. Als Besonderheit des Deutschen erweist sich, daß nicht nur die minderrelevanten Kategorien zurlicktreten, sondem ebenso eine relevantere Kategorie; die sich damals im Auf- und Ausbau befindende Aspektkategorie. Damit wird die Tempus- und Modusprofilierung von beiden Seiten her bedingt. Zum anderen wird deutlich, daß auch die Chronologie dieser diachronen Etappen die innere Struktur dieses Umbaus bestaitigt: Das Feld wird, um im Bild von Figur $4 \mathrm{za}$ bleiben, von hinten, d. h. von rechts nach links aufgerollt. Zuerst - bereits zu spattmhd. Zeit - werden die am wenigsten relevanten Kategorien nivelliert, d.h. sowohl in ihrer Allomorphie reduziert als auch in ihrem Ausdrucksverfahren justiert: Zu stark ins Zentrum des Verbs $\rightarrow$ geratene $\propto$ Kategorien werden wieder in die Peripherie ausgelagert. Diese Frage nach dem optimalen Ort und der optimalen Art der Materialisierung einer Kategorie bildet den dritten Schwerpunkt dieses Beitrags.

Im folgenden arbeiten wir uns von rechts nach links auf der Skala in Figur 4. Integriert wird dabei das in seiner Funktion bis dato nur unzureichend erforschte (und bei Bybee in diesem Zusammenhang nicht berücksichtigte) Phinomen der Flexionsklasse, die als wenig relevant bewertet und am rechten Pol der Skala situiert wird (s. Figur 4).

+ relevant

- relevant

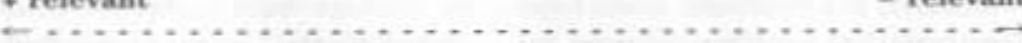

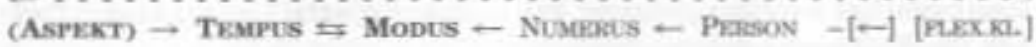

$\begin{array}{llllll}\text { Kap. } 3.6 & \text { Kap. } 3.5 & \text { Kap. } 3.4 & \text { Kap. } 3.3 & 3.2 & \text { Kap. } 3.1\end{array}$

Figur 4: Tempus- (und Modus-)Profilierung im Frühnhd.

\subsection{Flexionskt.ASsE: Nivellierung mit dem Resultat einer}

Zweiklassenopposition stark vs. schwach

Die drei schwachen ahd. Verbklassen ( $\sigma n_{-}, \epsilon n_{n}, c n-\lceil<j a n\}$-Verben) waren urspriinglich semantisch motiviert. Im Ahd. lassen sich noch gewisse Funktionsverteilungen erkennen, etwa indem die jan-Verben meist Kausative bildeten, die ên-Verben Durativa und Inchoativa ${ }^{5}$, die on-Verben Intensiva bzw. Iterativa. Diese einstigen Informationen der schwachen Flexionsklassen waren schon im Ahd im Schwinden begriffen. Hinzu kamen die im Infinitiv auf -an endenden starken Verben mit ihren sieben Ablautreihen, die bereits im Ahd. Aufspaltungen aufwiesen. Allerdings war es noch im Ahd. und Mhd. weitgehend möglich, jedem starken Verb die Ablautreihe, der es angehörte, zuzuweisen (aufgrund des Vokals bzw. seiner Lautumgebung) (s. Figur 5):

"Inchoativa wurden germ. durch die nan-Verben realisiert, die ahd in die enVerben eingegangen sind (vgl. Sonderegger 1979, S. 91).

\begin{tabular}{|c|c|c|c|c|}
\hline \multirow[b]{2}{*}{ Ahd. } & \multicolumn{3}{|c|}{ schwache Verben } & starke Verben \\
\hline & on: & en. & $\begin{array}{c}e n-(<j a n) \text {, } \\
\text { teilw. rückumlautend }\end{array}$ & $\begin{array}{c}\text { an } \\
\text { 7-10 AL-Reihen } \\
\text { AL-Reihe erschliefbar }\end{array}$ \\
\hline Mhd. & \multicolumn{3}{|r|}{ rückumlautend) } & AL-Reihe erschliefbar \\
\hline Nhd. & \multicolumn{3}{|c|}{$(+6$ Ausnahmen mit e/a-Wechsel $)$} & $\begin{array}{l}\text { 40-50 Vokalaitemanzen, } \\
\text { Vokalwechsel arbitrair }\end{array}$ \\
\hline
\end{tabular}

Figur 5: Nivellierung der Flexionsklassen vom Ahd. > Nhd. (ohne Prateritopriksentia; $\mathrm{AL}=$ Ablaut)

Flexionsklasse manifestierte sich im Ahd also bereits im Infinitiv durch distinkte Endungen, meist aber auch in den anderen finiten und infiniten Formen. Im Mhd. fallen die schwachen Klassen bis auf eine Untergruppe der jan-Verben (Rückumlautverben, s.u.) dem phonologischen Wandel der Nebensilbenabschwaichung zum Opfer. In Teilen ihres Paradigmas - z. B. Infinitiv und Prissens - werden sie den starken Verben ahnlicher, -an, - en, -en und $-0 n$ werden zu -en nivelliert; auch die Personalendungen erfahren starke Vereinheitlichungen. Wurzelintem realisierte Flexionsklassenmarkienung (Ablaut, Umlaut) bleibt (zunächst) erhalten. Bis heute trennt das Ablautverfahren die starken von den schwachen Verben. Innerhalb der schwachen Verben bleibt im Mhd. eine größere Gruppe der rückumlautenden Verben (mit ca. 220 Mitgliedern, vgl. Wegera/Solms 2000) erhalten, de ren Merkmale in umgelauteten Infinitiv- und Indikativ Prïsens-Formen sowie in nichtumgelauteten Präteritalformen bestehen. Die Opposition $+1-$ Umlaut diente also dem (relevanten) Tempusausdruck (s. Figur 6),

Erst im Verlauf des Frïhnhd. werden auch die Rückumlautverben abgebaut - bis auf die sechs Relikte brennen, rennen, kennen, nennen, sen. den, wenden, die zwei Gruppen von Reimwörtern bilden; die kleinere

\begin{tabular}{|c|c|c|}
\hline \multicolumn{2}{|c|}{ mittelhochdeutsch } & frïhnhd./nhd. \\
\hline $\begin{array}{l}\text { Inf., } \\
\text { Priis.Ind } / \text { Kond. } \\
\text { hier. 1.Sg.Prias. } \\
+ \text { Umlaut }\end{array}$ & $\begin{array}{l}\text { Prat.Ind. (flektier- } \\
\text { tes Part. II); } \\
\text { hier. 1.Sg Prit. } \\
\text { - Umlaut } \\
\end{array}$ & $\begin{array}{l}\text { Gesamtparadigma: Ausgleich nach den } \\
\text { +Umlaut-Formen } \\
\text { hier: 1.Sg.Prüs./Prat. } \\
\text { (Umlaut lexikalisiert) } \\
\end{array}$ \\
\hline $\begin{array}{l}\text { horne } \\
\text { kilisse } \\
\text { filiege } \\
\text { trōume } \\
\text { setae }\end{array}$ & $\begin{array}{l}\text { horte } \\
\text { kuste } \\
\text { fuocte } \\
\text { Eroumie } \\
\text { sazte }\end{array}$ & 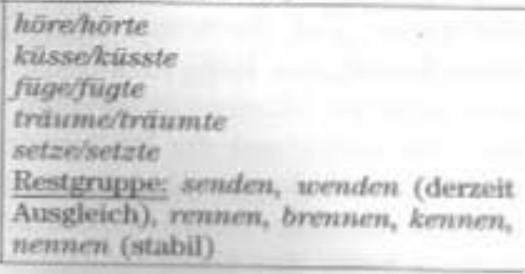 \\
\hline
\end{tabular}

Figur 6: Der Ausgleich der Ruckumlautverben im Fruhnhd. 
Reimgruppe sendenhenden ist derzeit im Ausgleich begriffen (sandte) wandte neben sendete/wendete). Die Stabilität der verbleibenden Vierergruppe läßt sich u.E. durch die Art des Vokalwechsels erklären: $\odot / a$-Wechsel zwischen Präsens und Prateritum findet sich nicht selten anch bei starken Verben (vgl. helfen/half) und könnte sekundär mit diesem Ablautmuster identifiziert worden sein.

Beim Abbau der Rückumlautverben handelt es sich um einen analogisch und damit morphologisch motivierten ProzeB, der deutlich von phonologisch motivierten Prozessen unterschieden werden muB. Er vollzieht sich vom 13/14. Jhd. bis zum Beginn des 16. Jhds, im Oberdeutschen. Im Mitteldeutschen beginnt er erst im 15/16. Jhd. mit bis heute ertaltenen Keineren Beständen. Der nhd. Zustand ist Ende des 16. Jhds, erreicht. Bei besonders frequenten, ela-alternierenden Verben wie setzen, schenken, stellen zieht sich dieser Ausgleich bis weit ins 18. Jhd. hinein (Moser/Stopp (Hgg.) 1988, S. 492-496). Dabei werden die Formen mit Umlaut generalisiert (vgl. nhd. küssen, trüumen, hören, fügen, setzen etc.), was mit deren höherer Frequenz (Indikativ Präsens) zu begründen ist. Dieser Abbau der Rückumlautverben wird bei der Behandlung der Modus-Kategorie wieder aufzugreifen sein. Beide Schube - sowohl der phonologisch motivierte im Mhd. als auch der morphologisch motivierte im Fruhnhd. - werden swiderstandslose durchgefuhrt, d. h. die (damals bereits opake) Kategorie Flexionsklasse wird nicht restituiert.

Durch die Reduktion des Spektrums an Flexionsklassen vertiefen sich die Zäsuren zwischen den verbleibenden beiden GroBklassen: Vokalwechsel und Dentalsuffix schließen sich gegenseitig ans. Einerseits werden simtliche schwachen Verben zu einer Klasse nivelliert, wohin schließlich auch die synchron zwischen stark und schwach stehenden und damit als Zwischenklasse zu charakterisierenden Rückumlautverben (Vokalwechsel + Dentalsuffix) übergehen, andererseits werden die starken Verben durch die Zersplitterung des Ablautsystems und die Aufspaltung in ca. 40 Vokalalternamzen sttirkere, Insgesamt erfahren sie vom Mhd. zum Nhd. eine Reduktion um etwa $50 \% 339$ starken Verben im Mhd. stehen noch $169 \mathrm{im}$ Nhd. gegenuber, die jedoch, wie Augst (1975, S. 263) treffend und zurecht bemerkt, sheute stiaker [sind], als es manchem Systemgrammatiker lieb sein kann-, und durch itre hohe Gebrauchsfrequenz gestiutzt werden. Hinzu kommt eine Reihe hochfrequenter, irregullarer Verben, die sich jenseits jeglicher Klassenzugehörigkeit befinden (z. B. gehen, stehen, sein, tun). Die Kleinklasse der Prateritopräsentia, gefestigt durch semantischfunktionale Merkmale (Modalitait), erfährt zwar eine quantitative Reduktion (nichtmodale Verben werden schwach), doch hat sich nicht zuletzt dadurch heute eine kleine Gruppe von sechs markanten und extrem frequenten Verben stabilisiert (wollen, sollen, können, diăfen, mögen, müis- sen, auch wissen) ${ }^{6}$, die bei genauerem Hinsehen wieder eine Zwischenposition zwischen stark und schwach einnehmen, indem sie ihr Pratteritum einerseits mit Dentalsuffix, andererseits (i.d.R.) aber auch mit (sekundir und irregulär erworbenem) Vokalwechsel bilden (vgl. kann/konnte, will wollte etc.). Weitere Klassenmerkmale treten hinzu.

Als eine sSackgasse der sprachlichen Evolution * könnte eine sich im Frihnhd, anbahnende Annäherung zwischen starker und schwacher Flexion bezeichnet werden, die in dem sog. epithetischen - $e$ in der 1 . und 3 . Person $\mathrm{Sg}$. Pratteritum der starken Verben bestand (ich/er flohe, ritte, sahe). Am plausibelsten ist die Erklianng durch Analogie zu den entsprechenden schwachen Formen (ich/er machte). Imsiepen (1983) zufolge erreicht dieser sich im 15. Jhd. anbahnende Wandel seinen Hohepunkt zu Ende des 17. Jhd., um im 18. Jhd. wieder komplett abgebaut zu werden bis anf die Form wurd-e als letztes Relikt.

Als Fazit ist festzuhalten: Insgesamt erfahrt das Flexionsklassensystem schon zu mhd. Zeit starke Reduktionen mit dem Resultat, daß die wenigen verbleibenden Klassen in umso stäkeren Kontrast zueinander treten. Insofem könnte durchaus von einer sukzessiven Zweiklassenprofilierung gesprochen werden, sieht man hier von den Präteritopräsentia ab, die eine Zwischenposition einnehmen. Zwar verschiebt sich seit frühnhd. Zeit die Opposition stark/schavach insbesondere in quantitativer Hinsicht zugunsten der schwachen Verben, doch ist ein Restbestand von weit uber 100 starken Verben, da zum Grundwortschatz gehörig, anch für die Zukunft gewilhrieistet (zum diachronen Ab-, Um- und Ausbau der Verbklassen im Deutschen siehe eingehend Dammel 2003).

3.2 Preson: Nivellierung von Person bei Stärkung von Numrrus

Figur 7 gibt einen Uberblick über die drei wichtigsten Nivellierungen der Kategorie Person. Bei deren Besprechung folgen wir der Chronologie des Abbaus, der bei der 3. Person Plural beginnt.

\begin{tabular}{|c|c|c|c|c|c|}
\hline \multirow[b]{2}{*}{$\begin{array}{l}\text { Flexionsklasse/ } \\
\text { Kategorienkombination }\end{array}$} & \multicolumn{3}{|c|}{ 2. Pers. Sges } & \multicolumn{2}{|c|}{ 3. Pers. PL. } \\
\hline & $\begin{array}{l}\text { st. V/I } \\
\text { Ind Prat. }\end{array}$ & $\begin{array}{l}\text { Prat_Pras/ } \\
\text { Ind.Prise }\end{array}$ & sonst & $\begin{array}{l}\text { st. V., sw.V/ } \\
\text { Ind Pras. }\end{array}$ & sonst \\
\hline Allomorphe mhd. & $\begin{array}{l}{[\mathrm{PL}-\mathrm{Al}} \\
\mathrm{UL}+-\mathrm{e}]\end{array}$ & $|-t|$ & $\mid$-st] & [-ent] & (-en) \\
\hline Morphe nhd. & \multicolumn{3}{|c|}{ |st| } & \multicolumn{2}{|c|}{ |-en] } \\
\hline
\end{tabular}

Figur 7: Reduktion der Allomorphie fur die Kategorie PErsoN

* Wollen, das unsprüinglich kein Priteritoprisens ist, hat sich der Klasse anteschlossen, nhd. bratichen kommt derzeit hinxu. 
3.2.1 Endung der 3. Person Plural Indikativ Praisens [-ent] > [-en\}

Bereits das Mhd. beginnt damit, die Endung in der 3. Pers. PL. Pràsens -ent der starken und schwachen Verben morphologisch $\mathrm{zu}$-en zu vereinfachen (sie geb-ent > sie geb-en), wobei intraparadigmatischer Ausgleich (nach dem Synkretismus-Muster des Prat. Ind. und Konj: L $=3$. Pers. $\mathrm{PL} .=-e n$ ) wahrscheinlich ist. Als Resultat ist ein homogeneres Pluralparadigma zu konstatieren (durchgehender Synkretismus in der 1./3.PL). Diese Personen-Nivellierung verschärft die Numerus-Opposition, da der Plural in sich homogener wird und -en im Sg. nicht vorkommt:

$\begin{array}{llll} & \text { Ahd. }> & \text { Mhd. }> & \text { Fruhnhd. } \\ \text { 1. PI. } & \text { gebamēs } & \text { geben } & \text { geben } \\ \text { 2. PL. } & \text { gebet } & \text { gebet } & \text { geb(e)t } \\ \text { 3. P1. } & \text { gebant } & \text { gebent } & \text { geben }\end{array}$

In den Dialekten geht diese Entwicklung weiter zu echten Einheitspluralen, wobei unterschiedliche Grundlagen gewählt wurden (z, B. im Schwabisch/Alemannischen die 3.PL. - (e) $n t-z u$ Genauerem s. SDS III, Karte 34). Als Konsequenz ist festzuhalten, daß die 3. Person Plural nun in allen Tempora und Modi eine Einheitsendung erhalt; die bisherige tempus- und modusabhăngige Personen-Allomorphie wird zugunsten einer NumerusProfilierung beseitigt, was gleichzeitig eine geringfugige Tempus- und Modus-Nivellierung bewirkt. Dies gilt auch - allerdings rein phonologisch motiviert - für die Nivellierung der Vokalunterschiede durch die Nebensilbenabschwăchung: Im Präteritum Plural Indikativ galten im Ahd. $u$-haltige und damit den Tempus and Modusausdruck unterstïtzende Personalflexive $(-u n,-u t,-u n)$, die mhd mit den entsprechenden Konjunktiv-sowie Präsens Indikativ-Flexiven homophon werden (als -en, -et, -en). Damit wird der Tempus- und Modusausdruck auf intro- oder binnenflektierende Verfahren konzentriert. und reduziert. Daruber hinaus erfahren diese beiden Kategorien Profilierungen in ganz anderem Ausmaß und von ganz anderer Qualität (s.u.), weshalb auf die nur unterstítzende Personen-Allomorphie verzichtet werden kann (vgl. aber bis heute die charakteristische - 0 -Endung in der 1./3.Sg. Präteritum Indikativ der starken Verben).

\subsubsection{Uniformierung der Endung (-st) für die 2. Person Singular:}

Eine einschneidende Veränderung im Sinne einer drastischen Uniformierung erfahrt die Personalendung der 2. Person $\mathrm{Sg}$, sowohl bei den starken Verben im Prateritum als auch bei den Praiteritoprisentia im Präsens.

\subsubsection{Von (du) wiurf-e zu (du) warf-st: 2. Person Sg. Präteritum starker Verben}

Additiv-segmentale Morphe scheinen Analogieprozessen wegen ihrer Materialitat und besseren Segmentierbarkeit stärker ausgesetzt zu sein als modulatorisch und damit abstrakter realisierte Morphe. So erklärt sich, daß die (im Westgermanischen entstandene) Sonderform in der 2. Pers. $\mathrm{Sg}$. Prät. der starken Verben (mhd. würfe) in einem ersten Schritt das additive st-Suffix aus dem Präsens (oder dem Prät. der schwachen Verben) ubernimmt und erst in einem zweiten Schritt den pluralischen $\mathrm{Ab}$ lautvokal, der uberdies noch umgelautet war, an den Vokal der 1./3. Pers. Sg. Prateritum angleicht und dabei den Umlaut aufgibt:

ahd. wurf- $t>$ mhd. würf-e $>$ mhd./fmhd. wïrf-est $>$ warf-(e)st $>$ nhd. warf-st

Gemăß Weinhold (1883) und Erben (1970) wird die alte Endung - $e$ im Ober- und Mitteldeutschen bereits ab dem 11/12. Jhd. durch -(e)st ersetzt (würf-e > würf-est); dagegen erfolgt der Wurzelvokalausgleich (wï̌rf-est $>$ warf-(e)st) enst zu Ende des 14. Jhds.

Mit der Aufgabe dieser charakteristischen Personen-Allomorphie wird Person nivelliert und Numerus profiliert: Wie Figur 8 zeigt, wird durch die Vereinheitlichung des Ablautvokals im Pratteritum Singular eine scharfe Numerusopposition aufgebaut; genau genommen entsteht erst jetzt ein eigentlicher priteritaler Numerusablaut (wie er in den nordgermanischen Sprachen von alters her bestanden hat). Das Flexiv -st entwickelt sich zwar (in der natürlichkeitstheoretischen Terminologie, vgl. Wurzel 22001) durch sein Eindringen in andere Klassen zu einem sog. ubberstabilen Marker, doch erweist sich diese *Uberstabilitä $\star$ bei genauerem Hinsehen als Schwächungserscheinung: Wie in Abschnitt 2. ausgeführt, korreliert ein hoher Grad an Allomorphie, mit hoher Relevanz (auch Frequenz) der betreffenden Kategorie. Die Entstehung überstabiler Marker, d.h. der Abbau von Allomorphie zeugt umgekehrt von geringer kategorieller Relevanz. Die Uniformierung kann das endgültige Schwinden dieses Markers zwar aufhalten (vgl. auch das Genitiv-s bei engl. und skand. Substantiven), doch ist sie u.E. als Zeichen des Abbaus, als eine Etappe im NivellienungsprozeB der betreffenden Kategorie zu bewerten. Es erfordert höheren kognitiven Aufwand, fur den Ausdruck ein und derselben Kategorie mehrere Allomorphe bereitzuhalten.

In Figur 8 wurde der erst im nächsten Abschnitt $z u$ thematisierende pratteritale Numerusausgleich integriert, da er in direktem Zusammenhang mit der Personennivellienung steht. Die Nivellierung der 2. Person Sg. bahnt sich bereits zu spätmhd. Zeit an und ist im Frühnd. vollzogen, wobei dieser Prozeß sich in der 3. Ablautreihe besonders lange hinzieht. 


\begin{tabular}{|c|c|c|c|c|}
\hline \multicolumn{2}{|c|}{ Prat. Ind. } & Mhd. & Frïhnhd. & Nhd. \\
\hline \multirow[t]{3}{*}{$\mathrm{Sg}$} & 1. & bant & band & band \\
\hline & 2. & bünd-e (> bind-ext) & band-est & band-est \\
\hline & 3. & bant & band & band \\
\hline \multirow[t]{3}{*}{ PI. } & 1. & bund-en & buend-en & band-en \\
\hline & 2. & bund-et & bund-et & band-et \\
\hline & 3. & bund-en & buond-en & band-en \\
\hline & & $\begin{array}{l}\text { kein ldares System } \\
\text { (Plural relativ } \\
\text { homogen) }\end{array}$ & $\begin{array}{l}\text { Prasocses. } \\
\text { Nivellierung > } \\
\text { Nusmats. Profilierung } \\
\text { (Numerusablaut): } \\
\text { band vs. bunden }\end{array}$ & $\begin{array}{l}\text { Nuswars-Nivellierung } \\
\text { (Ausgleich) > Traurts } \\
\text { Profilierung } \\
\text { binden vs, band vs. } \\
\text { gebunden }\end{array}$ \\
\hline
\end{tabular}

Figur 8: Personennivellierung > Numerusprofilierung (fmhd.); Numerusnivellierung (prateritaler Numerusausgleich) $>$ Tempusprofilierung (nhd)

Linger danert der Nivellierungsproze $B$ anch bei den Prateritopraisentia, die im folgenden Absatz zur Sprache kommen.

3.2.2.2 Von (du) darf-t zu (du) darf-st: 2 . Person Sg Präsens der Prateritoprïsentia

Deutlich spatter als bei den Praterita der starken Verben vollzieht sich die Uniformierung der 2. Person Sg. $-t \rightarrow-s t$ im Praisens der Prateritopräsentia, bedingt sowohl durch đie höhere lexikalische (Modalverben) als auch kategorielle Frequenz (Indikativ Prisens). Fur den Frequenzfaktor spricht des weiteren, daß sich dieser Umbau, wie die folgenden Zahlen (nach Best 1983) zeigen, als stark einzelverbgebunden erweist:

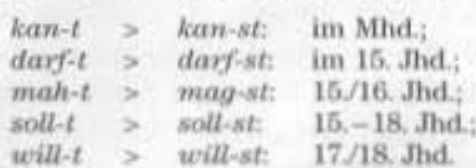

Da sich bei den beiden anderen Präteritopräsentia (müssen und wissen) wegen ihres wurzelfinalen -[s] Segmentierungsprobleme in der 2. Person $\mathrm{Sg}$. ergeben, blieben sie hier unberücksichtigt. Sie können jedoch als $\mathrm{Mu}$ ster diesen Umbau gestutzt haben.

Die beiden hier beschriebenen Nivellierungen der Kategorie Person tragen indirekt auch zur Flexionsklassen-Nivellierung bei: Indem Klassenunterschiede von der minderrelevanten Kategorie Person entkoppelt werden, beschrainken sie sich zunehmend auf relevantere Kategorien wie Modus und Tempus. Die Klassenunterschiede werden so quantitativ geringer. aber qualitativ deutlicher.
3.3 NUMERUs: Schwächung von NuMErus bei Profilierung von TEMPUS

\subsubsection{Der prateritale Numerusausgleich}

Der pratteritale Numenusausgleich bildet den wichtigsten AusgleichsprozeB im Frühnhd. und markiert die Grenze zum Nhd. Auch wenn dieser Proze $B$ besonders in den Ablautreihen I, IV und V eine gewisse phonologische Fundierung enthalten kann, so ist er in der Hauptsache als morphologisch motivierter AusgleichsprozeB zu betrachten. Fir die im einzelnen komplizierten, of in verschiedene Richtungen tendierenden, ablautklas senabhängigen und sich äber viele Jahrhunderte enstreckenden Ausgleichsprozesse sei auf die einschlagige Litenatur verwiesen (Strömberg 1907, Granmark 1933, Alm 1936, Solms 1984, Chirita 1988, Nübling 1999), Sie betreffen ausschlieBlich die Stufen 2 und 3 (fette Umrandung in Figur 9). Als Resultat ist die Reduktion von vier auf drei Ablautstufen und gleichzeitig die totale Nivellienung der ablautenden Numerusopposition im Priateritum zu konstatieren. Dies hat zur Folge, daß sich der Ablaut nur noch auf die Tempusdifferenzierung beschrinkt, damit also eine Ablauttemporalisierung stattgefunden hat (Augst 1975).

\begin{tabular}{|c|c|c|c|c|c|}
\hline$=$ & $\begin{array}{c}1 \\
\text { Inf., Prî̀s., } \\
\text { Konj. } 1\end{array}$ & $\begin{array}{c}2 \\
\text { Prā̄t. } \\
\text { Singular }\end{array}$ & $\begin{array}{c}\text { 3 } \\
\text { Prait. Plaral } \\
\text { Konv. II }\end{array}$ & ${ }^{4}$ Part. Perf. & $\begin{array}{l}\text { Zeitraum } \\
\text { (circa) }\end{array}$ \\
\hline $\begin{array}{l}\text { II } \\
\text { IIIa } \\
\text { IIIb } \\
\text { IV } \\
\text { V }\end{array}$ & $\begin{array}{l}\text { rit-en } \\
\text { biet-en } \\
\text { bind-en } \\
\text { werg-en } \\
\text { stel-en } \\
\text { geb-en }\end{array}$ & $\begin{array}{l}\text { (reit) } \\
\text { bôt } \\
\text { bant } \\
\text { uarff } \\
\text { (stal) } \\
\text { (gap) }\end{array}$ & $\begin{array}{l}\text { rit-en } \\
\text { (lout-en) } \\
\text { (bund-en) } \\
\text { (unurf-en) } \\
\text { stat-en } \\
\text { gab-en }\end{array}$ & $\begin{array}{l}\text { ge-rit-en } \\
\text { ge-bot-en } \\
\text { ge-bund-en } \\
\text { ge-uorf-en } \\
\text { ge-stot-en } \\
\text { ge-geb-en }\end{array}$ & $\begin{array}{l}\text { ab 15. Jhd. } \\
\text { ab 15. Jhd. } \\
15 .-19 \text {. Jhd. } \\
\text { 15. }-19 \text {. Jhd } \\
\text { ab 13. Jhd. } \\
\text { ab 13. Jhd. }\end{array}$ \\
\hline $\begin{array}{l}\text { VI } \\
\text { VII }\end{array}$ & $\begin{array}{l}\text { far-en } \\
\text { slaffen }\end{array}$ & $\begin{array}{l}\text { fuor } \\
\text { slief }\end{array}$ & $\begin{array}{l}\text { fuonen } \\
\text { stief-en }\end{array}$ & $\begin{array}{l}\text { ge-faren } \\
\text { ge-slaf-en }\end{array}$ & \\
\hline
\end{tabular}

Anm.: Fettdruck: die sich im Frnhd. durchsetzende Al.Stufe

Klammer die im Frnhd. abgebaute Al-Stufe

Figur 9: Die mhd. Ablautreihen 1-5 und die Richtungen des fruhnhd. pratteritalen Numerusausgleichs in den Stufen 2 und 3 (vereinfacht)

3.3.2 Ausgleich der Numerusopposition im Prisens der starken Verben der Reihe II (kreucht $>$ kriecht $)$

Aufgrund lautgesetzlich regulärer Entwicklungen kam es im Präsens der Verben der 2. Ablautreihe zu der mhd. Vokalopposition -iu- [y:] vs, -ie[is] (ich kriuche, du kriuchst, er/sie kriucht vs, wir kriechen, ir kriechet. sie kriechent). Im Frihnhhd wird iu [y: $>$ eu [oi] diphthongiert und ie [iə] $>[$ it] monophthongient, womit eine Opposition - eu- [oi] vs. -ie- [i:] ent- 
steht. Dieser numerusbezogene Volcalwechsel wird im Frühnhd. restlos beseitigt zugunsten der ie-haltigen Form. Vordergrundig ist damit keine Tempus- oder Modusstiarkung verbunden, allenfalls insofern, als Vokalwechsel sich immer mehr zur exklusiven Markienung von Tempus und Modus der starken Verben entwickelt. Diesem praisentischen Numerusausgleich steht die bis heute tradierte sog. Wechselflexion bei über 50 starken Verben gegenaber (siehe hierzu Kap. 4.1).

3.3.3 Ausgleich grammatischen Wechsels: Numeruszilsur wird beseitigt (teilweise zugunsten einer Tempuszalsur)

Bei den meisten Verben wurde der grammatische Wechsel, der durch die 2. und 3. Ablautstufe verlief und damit die prateritale Numerusopposition an zentraler Stelle (in bzw. an der Wurzel) unterstitzte, restlos beseitigt: mhd. veriliesen - verib̈s - verturen - verioren > nhd. verlieren - verior verioren (Moser/Stopp (Hgg) 1988, \$166). Besonderheiten enthalten jedoch einige besonders gebrauchsfrequente Verben wie zichen und sein.

Bei ziehen (Ablautklasse 2) wurde der grammatische Wechsel zwar beibehalten, doch besteht im Prateritum die Besonderheit, daB das $-g$ - der 3 . (eliminierten) Ablautstufe auf die (generalisierte) 2. Ablautstufe ubertragen wurde $(z \hat{h} \rightarrow z 0 g$ wegten zugen bzw. gezogen). Die dadurch entstehende Opposition zwischen $-h$ - bzw. $\sigma$ (mhd. $-h->$ frnhd. $-\emptyset$-) im Prásens versus $-q$ - im Prateritum und Part. Perf. verschärft die Tempusopposition und trigt damit zur Tempusprofilierung bei: ziehen vs. zog/gezogen. Dieser ProzeB fuhrt gleichzeitig zu einem gegenaber dem Prisens merkmalhafteren (lingeren) Prateritumausdruck: Präsens [tsi:-] versus Präteritum [tso:k/tso:g-]. Zusammenfassend:

$$
\begin{aligned}
& \text { mhd. aiehen - zoh vs. (zugen) - gezogem } \\
& \begin{array}{ll}
>\text { nhd. zichen vs. zog } & \text { - gezogen } \\
\text { [tsi:(o)n] vs. [tso:k] } & \text { - [ga'tso:gon] }
\end{array}
\end{aligned}
$$

Eine ahnliche Temporalisierung des grammatischen Wechsels hat bei schneiden - schnitt/geschnitten, leiden - litt/gelitten und sieden - sott gesotten stattgefunden (Verstilikung der Tempuszilsur, auch durch die Vokalkirzang vor stimmlosem - $t$ - vgl. demgegenuber meiden - mied - gemieden).

Etwas komplizierter liegen die Dinge bei dem höchstfrequenten Verb sein, das schon von alters her (d.h. im Germanischen) suppletiv organisient ist, groBtenteils aber - was die Suppletivwurzel wes- betrifft - der 5. Al-Reihe angehört:

mhd. $\sin$ - (was) - wären - gesesen < giveernan

$>$ nhd sein - unur - grinesen
Hier hat eine starke Irregularisierung stattgefunden, die den ohnehin suppletiven Charakter des Verbs verstlirkt hat: Im Prateritum wurde die 3. AL Stufe mit $-r$ generalisiert (nhd. warhwaren), in die 4. Stufe ist jedoch tiber bisher ungeklairte Wege (schon seit fruhester schriftlicher Oberileferung) das -5- der 2. AL-Stufe gelangt (vorahd. "giveran > mhd/nhd. gevesen).

\subsection{Moous: Nivellierungen und Profilierungen}

Durch die Nebensilbenabschwachung hat der Modusausdruck insgesamt starke Nivellierungen erfahren. Dies betriff Konjunktiv I wie II.

\subsubsection{Schwächung von Moous bei den schwachen Verben durch} Ausweichen in die Konjunktivperiphrase (wïnde-Uimschreibung)

Die Nebensilbenabschwächung verursacht den Zusammenfall von Präteritum Indikativ und Konjunktiv II:

$$
\begin{aligned}
& \text { ahd. } \\
& \text { mhd. suochta Prat. vs. suochti Kond. II. } \\
& \text { (fruh)nhd. suchte Prat. and Konj. II. } \\
& \hline
\end{aligned}
$$

Durch den Indikativ/Konjunktiv-Synkretismus weicht der Konjunktiv in die syntaktische Konstruktion aus und damit in ein deutlich expandierenderes Verfahren.

34.2 Stärkung von Moous bei den starken Verben durch Schwächung von TEmpus bei den schwachen Verben: Abbau der Rückumlautverben

Unter 3.1 wurde bereits der Abbau bzw. Ausgleich der großen Gruppe der Rückumlautverben behandelt, die uber +/-UL eine Tempusopposition bildeten: +UL .Präsens vs. $-\mathrm{UL}$, Prateritum (mhd. hoeren - horte $\rightarrow$ nhd. hören, hörte; s. Figur 6). Aus natïrtichkeitstheoretischer Sicht kollidiert hier semantische Unmarkiertheit mit formaler Merkmalhätigkeit (umgelautetes Praisens) bzw. semantische Markiertheit mit Merkmallosigkeit (unumgelautetes Prateritum), Diese tempusmarkierende Umlautopposition wurde im Frnhd. abgebaut, womit die verbleibende Umlautopposition auf den Modus-Ausdruck eingeschränkt wurde (+UL, Konjunktiv r vs. -UL Indikativi), d. h, in der Terminologie der Natirlichkeitstheorie wurde vermehrte Transparenz hergestellt (one form $\rightarrow$ one function, vgl. Mayerthaler 1981): Ein bestimmtes Ausdrucksverfahren (Umlaut) wird kategoriell begrenzt. Mit seiner Enttemporalisierung hat der verbale Umlaut gleichzeitig eine verstarkte Modalisierung erfahren (s. den Rechtspfeil von Trapus $\rightarrow$ Mopus in Figur 4). Bei den Ruckumlautverben hat damit eine Degram- 
matikalisierung bzw, Lexikalisierung des Umlauts stattgefunden, d.h. heute muß er mit dem Lexem mitgelernt werden. Nicht zufallig erhält sich in der verbleibenden Restgruppe gerade die e/a-Modulation (brennen brannte): Sie kommt auch im Ablautsystem vor (z. B. helfen - half), war also im Gegensatz zu den Modulationen $\sigma / / o$, w/u etc. nicht zwingend als Umlaut interpretierbar. Hier zeigt sich, daß es nicht, wie oft vorgebracht wird, ${ }^{7}$ die sog, Obercharakterisierung, das doppelte präteritale Ausdrucksverfahren (Vokalwechsel + Dentalsuffix) als solches, gewesen sein kann, das zur Beseitigung dieser Klasse gefuhrt hat. ${ }^{8}$ Evidenz dagegen liefern die Prateritoprisentia, fast ausschlieklich hochfrequente Modalverben, die eine solche Obercharakterisierung bis heute kultivieren, diese sogar sekundiar, vermuttich uber analogische Verfahren, auf- bzw. ansgebaut haben" (vgl. willwoollte, darf/durfts, kann/konnte, weikhoustes außerhallo der Prateritopribentia auch denkt/dachte und bringtbrachte).

Mit dem Abbau der Ruckumlautverben konzentriert sich nun das Umlautprinzip fast nur noch auf die Markienung des Konjunktivs bei den starken Verben. Damit erweist sich bezuglich des Parameters Flexionsklasse etwas Wichtiges: Es ist die schwache Klasse, die der Profilierung der starken Klasse s'Tribut zollte, Tempusumlaut in der schwachen Klasse wird zugunsten von Modusumlaut in der starken Klasse aufgegeben (s. 3.4.3).

3.4.3 Profilierung von Mopus bei den starken Verben durch Grammatikalisierung des Konjunktivumlauts (Umlautmodalisierung)

Nach dem pratteritalen Numerusausgleich wird der Umlaut auch im Verbal. bereich $^{10}$ morphologisiert und grammatikalisiert: Nachdem ihm in manchen Ablautreihen (Figur 9) wie z. B. der zweiten und dritten durch den präteritalen Numerusausgleich zugunsten der 2. Ablautstufe die Ableitungsbasis entzogen worden war, hat sich der umlautende Konjunktiv weder als autonome Form erwiesen noch der Periphrase der schwachen Verben angeschlossen. Stattdessen war es das reine, abstrakte Umlautverfahren, das =konjunktivierte wurde: Der Konjunktivumlaut hat sich analogisch auf die sich durchsetzende 2. Ablautstufe ubertragen: ich büte $\rightarrow$ ich bate; ich triinke $\rightarrow$ ich tränke, ich halfe $\rightarrow$ ich halfe etc. Dies zeugt von fester Funktionalisierung. Da sich der Numerusausgleich gerade in der 3. Ablautreihe besonders split etabliert hat (oft erst im 19. Jhd.), ist

\footnotetext{
7 So etwa von Besch (1967, S. 317), Schirmunski (1962, S. 601), Bittner (1996).

* Allenfalls war dieses Verfahren zu aufwendig im Verhaltnis zur geringen Tokenfrequenx vieler dieser schwachen Verben.

- Darnit einher geht auch nicht zuletzt eine Klaseenprofitiening

16 Im Nominalbereich vollzieht sich seine Pluralgrammatikalisierung
}

voi einer langen Wirkung des morphologischen Konjunktivumlauts auszusehen.

Bei besonders tokenfrequenten Verben hat jedoch der alte Konj.II paradigmatische Autonomie erlangt: Trotz des priteritalen Numerusausgleichs wird die alte synthetische Konjunktivform im Fall von würfe, stiirbe, auch stünde etc, beibehalten." Wichtig ist dabei anch, daß sich der Konjunktivumlaut klassenüberschreitend auf einige hochfrequente schwache Verben analogisch ansgebreitet hat: haben $\rightarrow$ hatte; brauchen $\rightarrow$ brauchte $^{12}$ (daneben auch regulär brauchte). Hier wirkt der Faktor Gebrauchsfrequenz, denn sonst müBte der morphologische Umlaut bei allen schwachen Verben eintreten. Umgekehrt hat die (durchschnittlich) geringe Gebrauchsfrequenz der schwachen Verben zur Folge, daß der (in seinem Relevanzgrad ja gleich bleibende) Modus in die Syntax (Konjunktivperiphrase) verlagert wird (vgl. 2.3).

Indem sich der Konjunktivumlaut (in aller Regel) auf die neuen starken Pràteritalformen übertrilgt, bestaitigt sich auch hier wieder der Relevanz. unterschied zwischen Tempus und Modus: Tempus legt einen neuen Vokal fest, Modus folgt dem und projiziert darauf sein Umlautverfahren, d. h. Tempus ist Modus funktional wie formal hierarchisch ubergeordnet. Hier manifestiert sich deutlich der diagrammatische Ikonismus gemäß Bybees Konzept (s. auch Aikhenvald/Dixon 1998).

Der Abbau von Umlaut vollzieht sich zusammenfassend in folgenden Schritten:

(1) Abbau des Umlauts zur Markierung der 2. Pers.Sg.Prat. starker Verben: würfe > warfst;

(2) Abbau des Umlauts zur Markierung von Tempus bei schwachen Verben Beseitigung der Ruckumlautverben:

(3) Konzentration, Ausbau und Grammatikalisierung des Umlauts im Konjunktiv starker Verben (Umlautmodalisierung).

Die Chronologie dieser Einzelschritte legt nahe, daß erst nach dem Abbau des Tempusumlauts bei den Rückumlautverben der Weg bereitet war für die Umlautmodalisierung: Zunăchst wird das Umlautverfahren spassiv auf den Modusausdruck beschrïnkt (sieht man von der Wechselflexion ab), um nach dieser 1:1-Justierung von Ausdruck und Funktion produktiv zu werden und auf die neuen Präterita zuzugreifen (Umlautgrammatikalisierung).

I Dabei könnte auch eine Rolle spielen, daß der alte Konjunktiv dieser Verben, die alle im Pras. Ind. [e/e] als Stammvolal haben, deutlicher bzw, uberhaupt vom Indikativ differenziert ist: $[\mathrm{d} / \mathrm{e} ;-\mathrm{v}]$ statt $[\mathrm{e} / \mathrm{e}$ - $-\mathrm{c}]$

Der momhologische Konjunktivumlaut bei bruachen ist auch als Annahme eines prateritopraisentischen Klassenmerlanals und damit als ein Schritt zum Beitritt in diese Klasse au deuten, der das Verb funktional schon seit dem 18. Jh. angehourt. 
3.4.4 Stärkung von MoDus durch Enttemporalisierung von Konjunktiv I und II

Wegen ihrer fruheren Tempusgebundenheit werden Konjunktiv I und II auch Konjunktiv Prissens und Prateritum genannt. Im Laufe des Frühnhd. erfolgt eine Enttemporalisierung, so daß in bestimmten Funktionen Konjunktiv I und II heute austauschbar sind: Sie sagt, sie komme/käme/voürde kommen (s. Hartweg/Wegera 1989, S. 126, Wegera/Solms 2000, S. 1549). Der Konjunktiv versieht mehrere Aufgaben; wie das Beispiel zeigt, besteht in seiner Funktion der Redewiedergabe Austauschbarkeit in dreifacher Hinsicht, nicht jedoch in seiner Funktion als Konditional (wenn sie kämé) kommen würde/komme) oder als Optativ, In jedem Fall eignet ihm kein Tempusunterschied mehr. Damit hat sich Modus von Tempus entkoppelt.

Zusammenfassend wird deutlich, daß die relevante Moduskategorie $\mathrm{Ni}$ vellierungen wie Profilierungen erfahren hat: Nivellierungen bei den insgesamt weniger tokenfrequenten schwachen Verben, Profilierungen bei den insgesamt tokenfrequenten starken Verben. Dem Frequenafaktor kommt bei der Reorganisation des Kategorienausdrucks - neben dem Relevanzfaktor - eine zentrale Rolle zu.

\subsection{Trmpus: Profilierung}

Relevanter als Modus ist die Kategorie Tempus, die noch eher eine binnenflexivische Markierung (im Lexem) sverdient $\propto$, Genau hierfür wurde der Ablaut (bei den starken Verben) funktionalisiert, der eine weitaus effizientere und aufwendigere Tempusanzeige leistet als ein in seiner Qualität und Quantitat vorhersagbarer Umlaut: Der Ablaut ist heute kaum mehr vorhersagbar (je nach Analyse zwischen 40 und 50 verschiedene Alternanzen) und sorgt damit für einen weitaus allomorphreicheren, weniger regelgebundenen und gleichzeitig binnenflektierenden Tempusausdruck. Vor diesem Hintergrund ist die Enttemporalisierung des Umlauts bei den Rückumlautverben (zugunsten seiner Konjunktivgrammatikalisierung) nicht als tiefgreifende Tempusnivellierung zu betrachten. Die etwas weniger relevante Moduskategorie erhalt vorhersagbaren, motivierten Vokalwechsel, die relevantere Tempuskategorie dagegen weitgehend arbiträren, lingst demotivierten Vokalwechsel. Des weiteren entstehen Tempusprofilierungen =indirekt- durch mehnere der bereits genannten Kategoriennivellierungen:

- durch den Erhalt der 6.Endungen in der 1/3. Sg. Prät. Ind. der starken Verben (sah-e hat sich nicht durchgesetzt);

durch den prateritalen Numerusausgleich, der den Ablaut temporalisiert: durch die Modusnivellierung beim Ausweichen der schwachen Konjunktive in die Syntax (das Dentalsuffix wird zum Prateritalzeichen);

- durch die selektive Beibehaltung grammatischen Wechsels bei ziehen, leiden, schneiden und sein.

Als weitere einschneidende indirekte Tempusprofilierung kann die phonologisch motivierte und analogisch unausgeglichen gebliebene Aufspaltung und Zersplitterung der Ablautreihen bzw. Vokalaltemanzen bewertet werden, was insgesamt nicht nur eine immense Zunahme an pràteritaler Allomorphie bewirkt hat, sondem anch eine sArbitrarisienung = des Vokalwechsels: Wie bereits erwähnt, war es im Mhd. noch weitgehend möglich, ein starkes Verb aufgnund phonologischer Indikatoren seiner Ablautreihe zuzaweisen. Diese EnschlieBbarkeit ist heute weitestgehend erloschen. In anderen Fallen wurde diese Vokalaltemanzzunahme auch morphologisch verursacht durch Ausnahmen bagl. der Richtung des präteritalen Numerusausgleichs: So hat sich in Fall von werden - wurde - geworden ausnahmsweise die dritte statt, wie in dieser Reihe ublich, der zweiten Ablautstufe durchgesetzt (neben weiteren Irregularisierungen speziell bei diesem Verb). Aus den 5 Ablautreihen des Germanischen haben sich 7 bzw. - mit den Aufspaltungen in den Klassen I-III - 10 Ablautreihen im Ahd. gebildet. Heute verteilen sich die starken Verben gemaiß der DudenGrammatik (1998, S. 127) auf 39 Alternanzen (gemäB Augst 1975, S. 233 sogar auf 52), wobei auf 6 Gruppen mehr als 8 Mitglieder kommen, auf 17 Gruppen 2-7 Mitglieder, wahrend die verbleibenden 16 Alternanzen mit Einzelgingern besetzt sind (bei Augst sind es sogar 30).

Konträr dazu sind auch Uniformierungstendenzen feststellbar: So werden die ahd. Aufspaltungen der Ablautreihen I und II wieder uber Analogie ausgeglichen. Des weiteren verdient eine größere Gruppe von Verben aus unterschiedlichen Ablautreihen besondere Beachtung: Diese haben ihre angestammte Klasse verlassen, indem sie im Priteritum und im Partizip Perfekt festes, einheitliches o (lang oder kurz) generalisiert und sich damit der 2. Ablautreihe angenathert haben (z. B. melken - molk - gemolken, ebenso scheren, gären, webem, heben, fechten, flochten, schmelzen, glimmen, löschen, schwören usw. (s. Ebert u. a. 1993, \& M $113 \mathrm{ff}$.). Moser/Stopp ((Hgg.) 1988, \$172) zufolge verdoppelt dieses Einheits-Alternanzprinzip o (Prait.) - o (Part.Perf.) seinen Anteil an der Mense der starken Verben von $14 \%$ im Mhd. (= Ablautreihe 2) auf $28 \%$ gegen Ende der fruhnhd. Periode. Wie die Beispiele oben verdeutlichen, handelt es sich bei diesen Cbergaingern keineswegs um hochfrequente Verben aus dem Grundwortschatz, was die Vermutung nahelegt, daß auf diese Weise sschwächelnde- starke Verben stark bleiben konnten, indem sie sich in eine uniforme Reihe mit relativ hoher Typenfrequenz integrierten (s, auch Augst 1975). Bestätigung 
für diese Annahme liefert das Niederländische, das viel stärker als das Deutsche auf genau dieses Verfahren gesetzt hat und dadurch nicht nur weniger starke Verben an die schwache Klasse abgegeben, sondern einige schwache Verben in die starke Klasse (dieser Reihe II) befördert hat (zI Einzelnem s. Hempen 1988). ${ }^{13}$

\subsection{AsPEkT Nivellierung von AsPEkT zur Profilierung von TEMPUS}

Das Deutsche hat im Gegensatz zu den meisten seiner Schwestersprachen die Aspektkategorie nicht grammatikalisiert. Allerdings gab es dazu durchaus Ansátze, die jedoch bei genauerem Hinsehen keine Degrammatikalisierung, sondern eine sumgrammatikalisierung $s$ in Richtung und zugunsten einer Temporalisienung erfahren haben. Dabei handelt es sich vor allem um die ge-Prifigierung im Partizip Perfekt und um die Eingliederung von wenden + Infinitiv ins Tempussystem.

\subsection{1 >Perfektivitäk > ,Vergangenheitstempusc Deaspektualisierung} von ge-Bildungen

Das Pràfix ge-war urspringlich ein Derivationsaffix zum Ausdruck von Perfektivitat, also einer Aspektikategorie (Solms 1991), In dieser Funktion haftete es an sämtlichen Formen des betreffenden Grundverbs, so etwa bei gesehen im Sinne von setwas erkennen, erblickenc (dieses Verb wurde in alem. gse und lux. gesimn ssehen lexikalisiert), das in Opposition zu sehen sschauenc gestanden hat (heute lexikalisiert: hören - gehören, horchen - gehorchen, wahren - gevoahren etc.). Umgekehrt war das Prafix ge- bei inhärent perfektiven Verben wie finden, kommen, werden, treffen, bringen blockiert. Bereits im Ahd. wird es zur Bildung des Partizips Perfekt grammatikalisiert, womit es sich zunehmend aus den finiten Formen zurückzieht. Ist davon auszugehen, daß auch die Perfektperiphrase zunăchst und zuvörderst dem Ausdruck perfektiven Aspekts diente und dabei dem Praiteritum als Imperfekt gegenüberstand, so hat spattestens im Frühnhd. ein Funktionswandel zum festen Tempuszeichen stattgefunden. Noch sehr lange, z. T. bis ins 17. Jhd. hinein, bleibt das Partizip Perfekt der oben genannten perfektiven Verben unpräfigiert; erst daun wird diese Licke geschlossen (fruhnhd. ich habe funden > ich habe gefunden). Letztes Relikt ist worden in Passivperiphrasen (es ist gefunden worden). Damit hat die Aspektnivellierung zu einer Tempusprofilierung gefuhrt.

14 Evidenz findet sich auch im Luxemburgischen, das in einem $=$ Rettungsversuch fur seine letzten Verben mit starkem Prateritum die 2. Abluutreihe zur einzigen generalisiert hat (s. Werner 1900).
In direkter Verbindung mit der ge-Grammatikalisienung steht die Frage des Prateritumschwunds. Bei der Suche nach dessen Gründen wird immer wieder auf die Aspekthaltigkeit der frühen Perfektperiphrase hingewiesen, die schließlich in das Tempussystem ubergegangen ist und in Konkurrenz zum synthetischen Präteritum trat.

\subsubsection{Ingressivitat $>$ Futurc Deaspektualisierung von werden-Periphrasen}

Die mhd. und frünhd. Grammatiken beschreiben verschiedene, unter schiedlich stark grammatikalisierte Aspeltperiphrasen, bei denen das Auxiliar werden eine wichtige Rolle spielt. Man geht davon aus, daR Konstruktionen mit werden + Partizip Praisens anfänglich Inchoativitat markierten (ich werde fragend ich beginne zu fragenc). Vermutlich hieraus hat sich durch lautliche Reduktion und/oder reanalytische Prozesse die Konstruktion werden + Infinitiv entwickelt, die heute temporalisiert ist (ich werde fragen). Wiesinger (2001) beschreibt für das Bairische um 1400 stark grammatikalisierte Fügungen mit priteritalem werden + Infinitiv zum Ausdruck von Inchoativitait/Ingressivitut: er ward fragen ser begann zu fragenc. Periphrasen mit prisentischem werden dienten gemäß Wiesinger auch im Bairischen zunächst dem Ausdruck inchoativen Aspelcts. Als diese temporalisiert waren, wurde die priteritale Inchoativperiphrase isoliert und schlieBlich abgebaut. ${ }^{14}$ Heute ist werden + Infinitiv als vollgram. matikalisierter Futurausdruck fest in das Tempussystem integriert, ge nauer: Diese Periphrase hat das Tempussystem komplettiert.

\subsection{DixtHesE: Etablierung von Passivperiphrasen}

Nur am Rande sei erwähnt, daß sich im Verlauf der Sprachgeschichte auch ein periphrastisches Diathesesystem (Aktiv/Passiv) etabliert hat. Dabel hat sich innerhalb des Passivs eine aspektuelle Differenzierung herausgebildet, die Opposition zwischen dem sog. Vorgangs- und dem Zustandspassiv. Auch diese Grammatikalisierung ist hauptsächlich im Frühnhd. zu ver orten. Gegenwärtig schließen sich dem das sog. Dativ- oder Rezipientenpassiv an (er kriegt einen Zahn gezogen) sowie weitere, z.T. modalisierte Passivkonstruktionen (z. B. mit gehören).

14 Zu diesen und anderen Aspekticonstruktionen siche auch historische Grammati ken (z. B. Paul u.a. ${ }^{24} 1998, \$ 315,329$ ). Best/Kohlhase (1983) beschreiben im Zusammenhang des prateritalen Numerusausgleichs zwischen den Kopulas ward und wurde einen. Aspeltunterschied: ward inchoativi vs. wurde imperfektivi (s. auch Philipp 1980, S. 67). 


\section{Wechselflexion und Präteritopräsentia}

In die bisher beschriebene Gesamtentwicklung scheinen sich zwei Phănomene nicht integrieren zu lassen: die Wechselflexion und die Prateritopräsentia. Daher sollen sie in aller Kürze gesondert betrachtet werden.

\subsection{Wechselflexion}

Unter der Wechselflexion versteht man einen prïsentischen Vokalwechsel in der 2. und 3. Person Singular bei ca. 55 starken Verben. Dieser Vokalwechsel wurde durch $i$-haltige Flexive ausgelöst und geht sowohl auf die germanische Hebung als auch auf den primären und sekundären $i$-Umlaut zurück:

$\begin{array}{cccc}\text { Inf. } & \begin{array}{l}\text { Hebung } \\ \text { geben }\end{array} & \begin{array}{l}\text { ituL } \\ \text { fahren }\end{array} \\ \text { Sg. } & 1 & \text { gebe } & \text { fahre } \\ & 2 & \text { gibst } & \text { fahrst } \\ & 3 & \text { gibt } & \text { fahrt } \\ \text { PL } \quad 1 & \text { geben } & \text { fahren } \\ & 2 & \text { gebt } & \text { fahrt } \\ & 3 & \text { geben } & \text { fahren }\end{array}$

Damit werden minderrelevante Person- $\mathrm{Numerus-Kategorien} \mathrm{uber} \mathrm{binnen-}$ flexivische Verfahren ausgedruckt, was gemilB unseres dargestellten Konzepts einer diagrammatischen -Verzerrunge bzw. Kontraikonik gleichkommt. Die Wechselflexion war früher ausgepragter (auch bei kommen, rufen, hauen etc.), doch ist gegenwärtig kein Abbau feststellbar - es sei denn, starke Verben wechseln gesamtparadigmatisch in die schwache Flexion aber (melken, backen, gebären). Ansonsten ist die Wechselflexion (im Gegensatz zur Hebung im Imperativ) stabil, ja sie wird sogar bei den seltenen thergingen von der schwachen in die starke Flexion als enstes iubernommen, s. ugs. früg(s)t, käuf(s)t, fässt, bezähl(s)t etc. (s. Nubling 2001). ${ }^{15}$

is Man kann sogar argumentieren, die Wechselflexion sei im Frnhd ausgebant oder zumindest stabilisiert worden, indem sich die Verben mit Hebung exi dem Muster der Dimlautverten 1 ss 28 . Pers $8 \mathrm{~g}$ anschlossen. U rspründlich hatten diese Yerben auch in der 1 . Pers Sg. Prits. Hebung so das cin Numerussokal. wecherl hentand fond. ieh nime, du nimest, er nimet vs. Plunal ant e). Pleser

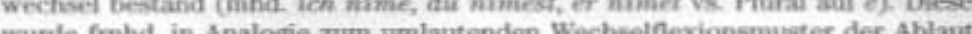

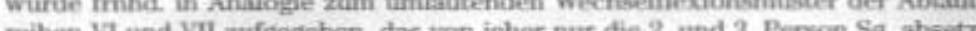
reihen 17 und Vil aufgegeben, das von jecher nur the 2 und 2 Person Se absetat (vgl. z B. Philipp 1980, S. 60). Hier hat sich also der fusionierte binnenflexivische Ausdruck fur Person-Numenus gegen den transparenteren reinen Numerus-Vokalwechsel durchgesetzx. Davzu kommt, daß der Numerus-Wechsel der 2 Al. Reihe ganz aufgegeben wurde (vgl. 3.3.2), so das die Wechselfexion (abgesehen von den Prateritoprasentia) zum einzigen prasentischen Vokalwechselmuster wurde. Das Verfiaturen, das die frequentesten Kategorienbündel 1. und 3. Pers. Sig. Prass. Ind. deutlicher differenziert, hat sich damit durchgesetat
Schaut man sich die Wechselflexionsverben genauer an, so erweist sich zweierlei: Zum einen handelt es sich um den frequentesten Bestand der (ohnehin durch ihre Gebrauchsfrequenz gestutten) starken Verben, vgl. werden, geben, nehmen, essen, fahnen etc, d. h. hier kommt der Faktor der Gebrauchsfrequena zum Tragen, der insgesamt Linksverschiebungen auf der Ausdrucksskala bewirkt (s. 2.3). Zu der lexikalischen Verbfrequenz tritt außerdem die kategorielle Frequenz: Die meistgebrauchten Paradigmenpositionen sind die Formen der 1. und 3. Pers. Singular im Präsens IndikativParadigma, die durch die Wechselflexion differenziert werden. Zum zweiten erweist der Vokalwechsel, daß sich hier eine Reduktion auf zwei Altemanzen vollzieht: Die stärkste Gruppe betrifft den e/i-Wechsel (36), die zweitstäkste den $a / a$-Wechsel (16) - also eher ablautahnliche, zumindest arbitrire Wechsel. Typische Umlautalternationen sind heute dagegen äußerst selten ( $a w / a u: 2$ Verben, o/o: 1 Verb; $u / i$ : kein Verb) und haben diachron tatsaichlich eine starke Dezimierung erfahren (vgl. noch die frühnhd. Wechselflexion bei kommen, rufen, hauen). Mit der Konzentration auf prinzipiell zwei Modulationsverfahren - in der 2. Ablautreihe wurde, wie in 3.3 .2 ausgefuhrt, die Wechselflexion ewie beseitigt - halt sich die Allomorphie in Grenzen, doch verzichtet das Deutsche nicht auf dieses fusionierende Person/Numerus-Ausdruckverfahren, das die anderen germanischen Sprachen (außer dem Isländischen und Luxemburgischen) abgebaut haben.

\subsection{Prăteritopräsentia}

Bisher haben wir die Präteritopräsentia vernachlässigt, was ihrer zentralen Position im System widerspricht. Die Prateritoprasentia haben zwar in der 2. Pers. Sg. die Uniformierung von $-t>-s t$ vollzogen (vgl. 3.2.2.2), doch in höherrelevanten Kategorien durchaus Profilierungen durchgefuhrt, wobei sie von unublichen Mitteln Gebrauch gemacht haben: Die vier Verben können, mögen, dïrfen, müssen haben im Präsens Plural sowie im Infinitiv eine nichtlautgesetzliche und bisher nicht zufriedenstellend geklärte Umlautung erfahren, die zu einer tiefen Numeruszasur gefuhrt hat:

\begin{tabular}{|c|c|c|c|}
\hline Inf. & & kônen & dïrfen \\
\hline $\mathrm{Sg}$ & $\begin{array}{l}1 \\
\frac{1}{3}\end{array}$ & $\begin{array}{l}\text { kamn } \\
\text { kamust } \\
\text { kamn }\end{array}$ & $\begin{array}{l}\text { darf } \\
\text { darfst } \\
\text { darf }\end{array}$ \\
\hline PL. & $\begin{array}{l}1 \\
2 \\
3\end{array}$ & $\begin{array}{l}\text { koomnen } \\
\text { kömnt } \\
\text { kômen }\end{array}$ & $\begin{array}{l}\text { dürfen } \\
\text { dürft } \\
\text { dürfen }\end{array}$ \\
\hline
\end{tabular}

Das Besondere ist, daß die Prăteritopräsentia ohnehin bereits einen Numenusablaut enthielten, diesen aber durch die Umlautung tiberlagert und 
damit die Numerusopposition sverschärfts haben. Wichtig ist also, daß Singular und Plural in keinem transparenten Umlaut-Ableitungsverhalinis stehen: Weder bei $a / \overline{0}$ noch bei $a / i$ handelt es sich um eine Umlautrelation (nur bei $w / u$ in milssen, wo nie Numerusablaut bestand und der Umlaut erst eine Numenusopposition geschaffen hat) ${ }^{16}$. Mit dem Ausbau der Numerusopposition im Präsens Indikativ, eines wichtigen Klassenumerkmals der Prateritoprisentia, wird in diesem Fall Flexionsklasse gestärkt. Schlieklich haben auch Tempus und Modus Profilierungen erfahren, Tempus durch diverse irreguläre Vokalwechsel, die zu dem an sich schwachen Prateritum hinzukommen (will/wollte, darf/durfte, kann/konnte, weiß/ wriste etc.), Modus durch den Umlaut (dürfe, könnte, wüßste etc.). Dabei handeln immer einige Mitglieder (vor allem sollen und wollen) manchen dieser Merkmale zuwider; der Klassenzusammenhalt beruht nicht auf transparenten Flexionsregeln, sondern eher auf Familienahnlichkeitsrelationen, was sich letztlich durch die hohe Frequenz der Modalverben (und damit lexikalische Autonomie ihrer Flexionsformen) erklären taßt.

\section{Fazit}

Primâr aufgrund jahrhundertelang wirksamer und akkamulierter phonologischer Entwicklungen wurde die Morphologie stark affiziert, zum einen durch mangelnde Markierungssubstanz (ansgelöst durch reduktive Prozesse), zum anderen aber auch durch Verzerrungen eines ausgewogenen Funktion-Form-Verhältnisses (ausgelöst durch assimilatorische Prozesse). Sehon zu mhd. Zeit bahnt sich ein grundlegender morphologischer Umbau an, der chronologisch bei den weniger relevanten Kategorien beginnt und sich im Laufe der Jahrhunderte in die jeweils höherrelevanten Kategorien arbeitet. Am Ende steht eine wJustierung « des Kategorienausdrucks bei einer gleichzeitigen Tempusprofilierung, die mit einer Konsequenz betrieben wurde, wie sie bei kaum einer anderen germanischen Sprache anzutreffen ist: Der vorrangig durch assimilatorischen Wandel bewirkten Ablautklassenzersplitterung wurde morphologisch kaum begegnet, vielmehr wurde diese zum Aufbau erhöhter Tempusallomorphie (passiv) genutzt Zum quantitativen Zuwachs an Allomorphie kommt ein qualitativer hinzu: Es entstchen vielfaltigere, arbiträrere (von der Lautumgebung entkoppelte) und damit weniger vorhersagbare Vokalaltemarzen, z.T. auch Konsonantenalternanzen (ziehen, leiden, stehen, gehen) bis hin zur Suppletion.

16 Nicht zufallig ist müssen Vorreiter bei der Pluralumlautung (s. z. B. Lahr 1987). Die Arunahme eines Numerusvolcalwechsels bedeutet hier eine morphologische Annahienung an die anderen Klassenmitglieder.
Gleichzeitig hat eine -Zentralisierung «, eine binnenflexivische Konzentration des Tempusausdrucks stattgefunden - etwa durch Abbau tempusabhalingiger Person/Numerus-Allomorphie (vgl. -ent $>-e n$ ) - und damit eine Entkoppelung interkategorieller Abhängigkeiten: Die Kategorien werden in ihrem Ausdruck tendenziell voneinander separiert. Zur Tempusprofilierung beigetragen haben nicht nur Nivellierungen minder, sondern auch höherrelevanter Kategorien: Aufkeimende Aspektderivation und Aspektkonstruktionen wurden abgebaut und in das Tempussystem integriert.

Dieser gesamte ProzeB darf nicht finalistisch oder teleologisch verstanden werden. Es handelt sich um chronologisch aufeinanderfolgende, lokale relevanz- und frequenzgesteuerte Optimierungen, die uber die Nivellierung vieler Verbalkategorien indirekt zur Profilierung von Tempus geführt haben (dies zeigen Figuren 4 und 10).

Auch der Modusausdruck hat eine Stärkung und Justierung erfahren, indem der Umlaut in der Person/Numerus/Tempus-Anzeige bei den starken Verben abgebaut wurde (wïrfe > warfst) und indem er sich aus der Tempusanzeige der Rückumlautverben zurückgezogen hat. Dies hat den Umlaut auf den Konjunktivausdruck beschränkt, der dort schließlich morphologisiert und nach dem präteritalen Numerusausgleich auf die neuen Prateritalformen ibertragen wurde. Allerdings findet er sich noch in 19 wechselflektierenden frequenten Verben zum Ausdruck von Person/Numerus $(2.3 . \mathrm{Sg})$, des weiteren auch von Tempus, da nur im (häufigen!) Prisens auftretend. Der Konjunktivausdruck erfolgt bei den starken Verben binnenflektierend (teilweise kommen spezifische Endungen hinzu), wobei weitaus weniger Allomorphie sowie qualitativ und quantitativ vorhersagbarer Vokalwechsel (Palatalumlaut) besteht (abgesehen von hochfrequenten Verben wie stüinde, würfe).

Auch das in seiner Funktionalität kaum gekläte Phànomen der Flexionsklasse wurde in den Blick genommen: Insgesamt hat eine Verringerung bei einer gleichzeitigen Polarisierung von Flexionsklassen stattgefunden. Am Beispiel der Rückumlautverben wurde deutlich, daß Tempusumlaut in der schwachen Klasse abgebaut, Modusumlaut in der starken Klasse aber ausgebaut wurde, d. h. die Markierung einer hoherrelevanten Kategorie wurde innerhalb der schwachen Klasse geschwicht und die einer weniger relevanten Kategorie in der starken Klasse gestärkt. Infolgedessen wird die Zasur zwischen starken und schwachen Verben vertieft: Modulative Verfahren sind allein starken Verben vorbehalten in Opposition zu rein additiven Verfahren der schwachen Verben. Als einzige Zwischenklasse hat sich die Gruppe der Präteritopräsentia semantisch gefestigt und formal profiliert, indem sie Verfahren beider Großklassen nutzt, doch was Vokalwechsel angeht, in von den starken Verben formal und funktional abweichender Weise. 


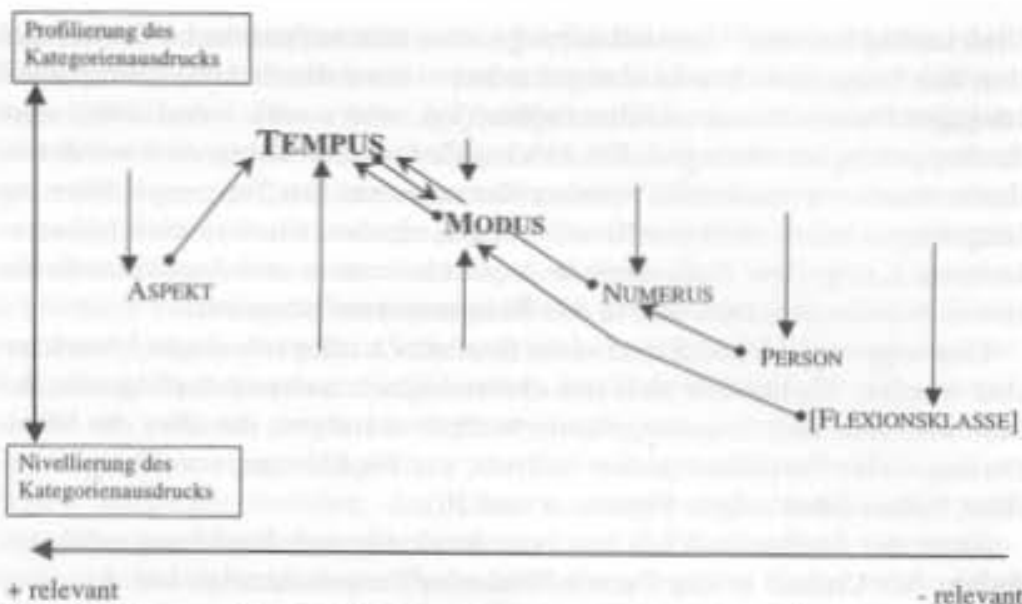

Figur 10: Kategorielle Profilierungen und Nivellienungen im Oberblick

Als zentral hat sich die Rolle der Relevanz erwiesen sowie die der lexikalischen und kategoriellen Frequenz. Damit erfahrt das Relevanzkonzept, das synchron anhand eines umfassenden Sprachvergleichs entwickelt wurde, Bestitigung durch die Diachronie des Deutschen (s. die zusammenfassende Obersicht in Figur 10).

\section{Literatur}

Abraham, W. 2001: Pratentumschwund und Diskursarammatik. Amsterdam, Phil. adelphia.

Aikhenvald, A. Y, a. R. M. W. Dixon 1998: Dependencies between gnummatical systems, Language 74, S. 56-80.

Alm, E. 1986 Der Ausgleich des Ablauts im starken Prateritum der ostmitteldeutschen Schriftidialekte, Uppsala.

Augit, G. 1975 . Whe stark sind die starken Verben? Oberlegungen zur Sublikssifizierung der nhd, Verben, in: G. Augst, Untersuchungen zum Morpheminventar der deutschen Gegenwartssyrache, Tubingten, S. 231-281

Besch, W. 1967: Sprachlandschaften und Sprachauspleich im 15. Jahrhundert. Studien zur Erforschung der spatmittelhochdeutschen Sclureibdialekte und zur Entstehung der neuhochdeutschen Schrifsprache, Muncher

1979. Zur Bestimmung von Rezularitaten bei den sprachlichen Ansgleichsvor: gaingen im Priłheuhochdeutschen, ZfdPh 98 Sondertheft, S. 130-150.

1980: Fruhneuhochdeutsch, in: Lexikon der sermanistischen Linquistik, Tubinthen, S. $585-597$.

-, u.a. (Hige) 19982000 (2. Aunlage): Sprachugrschichte. Ein Handbech zur Geschichte der deutschen Sprache und ihrer Erforschung. 2 Bde., Berlin, New York, [Darin: Einige wichtige Oberblicksartikel zum Frnhd, v, a. in Bd. II, Artikel $\mathrm{Nr} .110-123$
-, u. K.P. Wegera (Hgg) 1987: Fruhneuhochdeutsch, ZfdPh 106, Sonderhet.

Best, K.H 1983: Zum momhologischen Wandel einizer deutscher Verben. irt Besf Kohlhase (Hog), Exalate Sprachwandelforschiung Theoretische Beiträge, statistische Analysen und Arbeitsberichte, Gottingen, S. $107-118$

2. I Eohllhase 1963: Der Wandel von ucard zu vrurde, in: Bent/Kohlhase (Hige) Bralte Sprachwandelforschung. Theoretische Beitrage, statistische Analysen und Arteitsberichte, Gotringen, S. $91-102$.

Bittner, A. 1906 Starke sechwache- Verben und schwache intarios Verben. Deutsche Verthexion und Naturlichkeit, Tubingen.

Bogner, L. 1989: Zur Entwicklung der periphrastischen Futurformen im Frutunhd, ZfdiPh 108, S. 56-85.

Bybee, 1. 1985: Morphology, A study of the relation between meaning and form, Amsterdam.

- 1994: Mompological universals and change, in: R. E. Asher (Hg), The encyclopedia of language and linguistics, vol. 5, Oxford, S. 2557-25e2.

4. W Pagliuca 1987: The evolution of future meaning in A. Giacalone Ramat, 14. (Hoge), Papers from the $7^{\text {th }}$ international conference on historical linguistics, Amsterdam, S $109-122$

-. et al. 1994: The evolution of grammar. Tense, aspect, and modality in the languages of the world, Chicago.

Chirita, D, 1988: Der Ausgleich des Ablauts in starken Prateritum im Fruhneuhochdeutschen, Frankfurt

Damumel, A. 2003: Flexionsklassen Fumktionalitat oder reine Allomorphie? Zu AbUm: und Ausbau von Verbklassen im Deutschen. Magisterarbeit, Eniversitar Maing

Diewald, G. 1997; Grammatikalisienung, Eine Einfuhning in Sein und Werden grammatischer Formen, Tubingen.

Duden-Grammatik der deutschen Gegenwartseprache. Duden Bd.4, Mannheim "1998.

Ebent, R. P, u. 1983: Früheruhochdeutsche Grammatik, Tubingen

Frben, J. 1970. Frihneuhochdeutsch, in: L. E. Schmitt (Hg.), Kurser Grundriß der germanischen Philologie bis 1500 , Bd. 1: Sprachgeschichte, Berlin, S. 386-440.

Granmark, 0.8. 1933: Die Ansgleichung des Ablauts im starken Prateritum des rheinfrankischen Schrifttialekts. Ein Beitrag zur Formenlehre des Fruhneuhochdeutschen, Malmo.

Hartweg, F, u. K.-P. Wegera 1989: Früneuhochdeutsch. Tubingen (Germanistische Arbeitshefte 33)

Heine, Bernd 1095: On the German werden future, in: W. Abraham et al. (Hgg.) Discounse, grammar, and typology, Amstertam, 8. 119-138.

-. u. T. Kuteva 2002 . World lexicon of grammaticalization, Camdridge.

Hempen, U. 1988: Die starken Verten im Deutschen und Niederiandischen. Diachrone Morphologie, Tubingen.

Hoffmann, W, a. H.J. Solms 1987: Zur Flexion der starken und schwachen Verben, Zfdiph 106, Sonderhen, S. 37-69.

Hopper, P, I. E Traugott 1993: Grammaticalization, Cambridtec

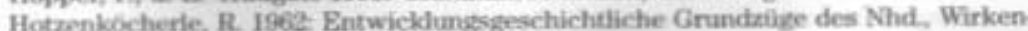
des Wort 12, S. 321-331

Imsieges, Hrike 1053: Die e. Ppithese bel starken Yerten im Deutschen, in: Pest Kohlliave (Heg), Erakte Sprachwandelforschung. Theoretische Beiträge, statistieche Anabsern und Arbeitsberichte, Gottinger, S. 119-141

Kem, P, II. H Zutt 1907 ; Geschichte des deutsohen Fexionssystems, Tubingen.

Kopecke, K.M. 1999: Prototypisch starke und schwache Verben der deutschen Gegenwartssprache, in- M. Butt a. N. Fuhrhop (Hgg.), Variation und Stabilităt in der Wortstniktur. Untersuchungen zu Entwicktung, Erwerb und Varietalten des 

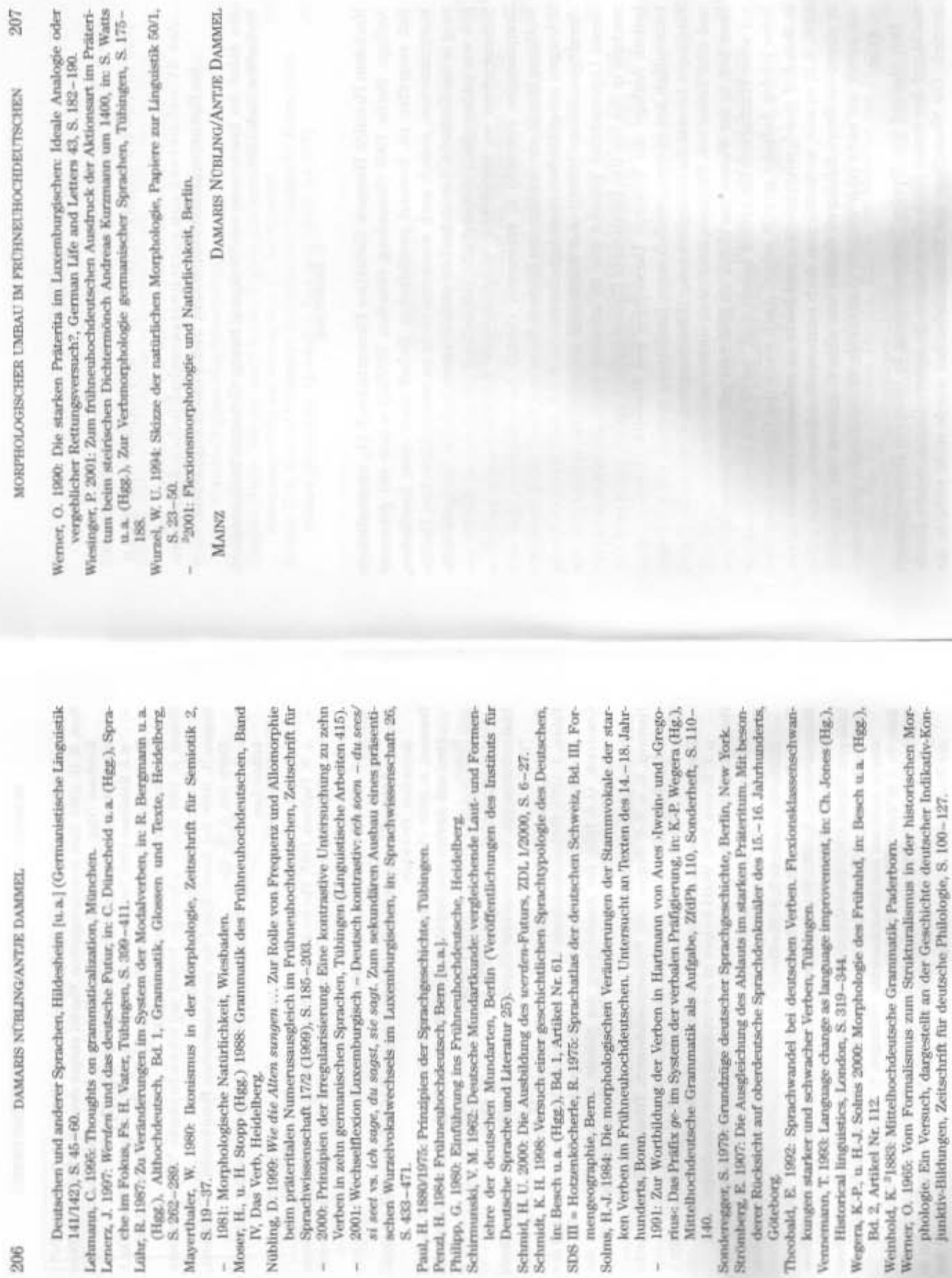